\title{
SOME RESULTS IN THE LOCATION OF THE ZEROS OF LINEAR COMBINATIONS OF POLYNOMIALS
}

\author{
BY \\ ZALMAN RUBINSTEIN ( $\left.{ }^{1}\right)$
}

We study here the location of the zeros of linear combinations of polynomials of the form $f(z)-\lambda g(z)$, where $f(z)$, and $g(z)$ are arbitrary polynomials with complex coefficients and $\lambda$ is a complex number. It is known [3] that this question is closely connected with the study of the zeros of polynomials of the form $(z-\alpha)^{n}-\lambda(z-\beta)^{r}$, which indeed is the main object of this paper.

We start with a particular case.

THEOREM 1. Let the polynomials $f(z)=z^{n}+\cdots$, and $g(z)=z^{r}+\cdots$, $n=2 r$, have zeros in the circles $|z-a| \leqq r_{1}$ and $|z-b| \leqq r_{2}$, respectively, then all the zeros of the polynomial

$$
f(z)-\lambda g(z)
$$

are in the union of the $n$ circles

$$
\left|z-a-\frac{1}{2} \lambda^{2 / n}+\lambda^{1 / n}\left(a-b+\frac{1}{4} \lambda^{2 / n}\right)^{1 / 2}\right| \leqq\left(r_{1}+r_{2}\right)^{1 / 2}|\lambda|^{1 / n}+r_{1},
$$

where $\lambda^{1 / n}$ assumes all the nth roots of $\lambda$.

Proof. The equation $f(z)-\lambda g(z)=0$ can be replaced by Grace's theorem [3] by the equation $(z-\alpha)^{n}-\lambda(z-\beta)^{n / 2}=0$, where $|\alpha-a| \leqq r_{1}$, and $|\beta-b| \leqq r_{2}$.

Solving for $z$ we obtain

$$
z=\alpha+\frac{1}{2} \lambda^{2 / n} \pm \lambda^{1 / n}\left[(\alpha-\beta)+\frac{1}{4} \lambda^{2 / n}\right]^{1 / 2} .
$$

Denoting generically the region $|z-c| \leqq R$ by $C(c, R)$ we have

$$
\begin{gathered}
\alpha-\beta \in C\left(a-b, r_{1}+r_{2}\right) \\
\left(\alpha-\beta+\frac{1}{4} \lambda^{2 / n}\right)^{1 / 2} \in C\left( \pm\left(a-b+\frac{1}{4} \lambda^{2 / n}\right),\left(r_{1}+r_{2}\right)^{1 / 2}\right)
\end{gathered}
$$

hence

Received by the editors August 21, 1963 and, in revised form, March 11, 1964.

( ${ }^{1}$ ) This work was supported by the Air Force Office of Scientific Research. 


$$
z \in C\left(a+\frac{1}{2} \lambda^{2 / n} \pm \lambda^{1 / n}\left(a-b+\frac{1}{4} \lambda^{2 / n}\right)^{1 / 2},\left(r_{1}+r_{2}\right)^{1 / 2}|\lambda|^{2 / n}+r_{1}\right) .
$$

(2) follows since, by assumption, $n$ is an even number.

The result is sharp for $\lambda=0$, and for $a=b$.

For the general case we have

THEOREM $2\left({ }^{2}\right)$. Let $f(z)=z^{n}+\cdots, g(z)=z^{r}+\cdots, n>r$, have zeros in the circles $|z-a| \leqq r_{1}$ and $|z-b| \leqq r_{2}$, respectively. Then all the zeros of the polynomial $f(z)-\lambda g(z)$ are in the circle

$$
|z-a| \leqq r_{1}+d,
$$

where $d$ is the positive root of the equation

$$
d^{n / r}-M d-N=0
$$

with

$$
M=|\lambda|^{1 / r}, \quad N=|\lambda|^{1 / r}\left(|a-b|+r_{1}+r_{2}\right) .
$$

Proof. Consider the equation

$$
(z-\alpha)^{n}=\lambda(z-\beta)^{r}, \quad|a-\alpha| \leqq r_{1}, \quad|b-\beta| \leqq r_{2} .
$$

For $z_{0}$ satisfying $\left(z_{0}-\alpha\right)^{n}=\lambda\left(z_{0}-\beta\right)^{r},\left(z_{0}-\alpha\right)^{n / r-1}=\lambda^{1 / r}\left(\left(z_{0}-\beta\right) /\left(z_{0}-\alpha\right)\right)$. Let $d_{1}$ be a positive number satisfying

$$
d_{1}^{n / r}-M d_{1}-N>0 .
$$

For $\left|z_{0}-\alpha\right| \geqq d_{1},\left(z_{0}-\beta\right) /\left(z_{0}-\alpha\right)$ belongs to the circle $|z-1| \leqq|\alpha-\beta| / d_{1}$; hence

$$
\left|\lambda^{1 / r} \frac{z_{0}-\beta}{z_{0}-\alpha}\right| \leqq|\lambda|^{1 / r}\left(1+\frac{|\alpha-\beta|}{d_{1}}\right),
$$

but

$$
\left|z_{0}-\alpha\right|^{n / r-1} \geqq d_{1}^{n / r-1}>|\lambda|^{1 / r}\left(1+\frac{|\alpha-\beta|}{d_{1}}\right),
$$

for all $\alpha, \beta$ such that $|\alpha-a| \leqq r_{1}$, and $|\beta-b| \leqq r_{2}$. We get a contradiction, which proves that $\left|z_{0}-\alpha\right|<d_{1}$.

It is worthwhile to remark that if $M+N>1$ an estimate for the positive zero $d$ is the expression

$$
\frac{(n-r)(M+N)^{n / n-r}+r N}{(n-r)(M+N)+r N} \leqq(M+N)^{r / n-r} .
$$

For $M+N<1$ a bound for the same is $((n-r+r N) /(n-r M)) \leqq 1$. $[5]$.

${ }^{2}$ ) Theorem 2 was proved independently and by a different method by Mishael Zedek 
Different estimates can be obtained by means of estimates similar to those used in the proof of Theorem 2, which are sharp for $\lambda=0$ or asymptotically for $\lambda \rightarrow \infty$. We indicate some of them which are of a relatively simple form.

TheOREM 3. Let $f(z)$ and $g(z)$ be as in Theorem 2. All the zeros of the polynomial $f(z)-\lambda g(z)$ are in each of the following regions:

$$
|z| \leqq \frac{|a|-r_{1}}{d\left(|a|-r_{1}\right)-1}\left[\left(|b|+r_{2}\right) d+1\right]
$$

where $r>n, d=|\lambda|^{1 / r}\left(r_{1}+|a|\right)^{-n / r}$, and $d\left(|a|-r_{1}\right)-1>0$.

$$
|z-b| \leqq r_{2}+2 \operatorname{Max}\left[|\lambda|^{-(1 / r-n)},\left(|a-b|+r_{1}+r_{2}\right)^{n / r}|\lambda|^{-(1 / r)}\right],
$$

where $r=n k, k \geqq 2$.

$$
\left|z-\frac{\delta_{k} b}{\delta_{k}-1}\right| \leqq \frac{m+\left|\delta_{k}\right|\left(r_{2}+1\right)}{\left|\delta_{k}-1\right|}, \quad k=1, \cdots, n,
$$

where $n>r, w_{k}^{n}=\lambda, \delta_{k}^{n}=\lambda /(1-\lambda), k=1, \cdots, n$;

$$
m=\operatorname{Max}_{1 \leqq k \leqq n} \frac{1}{\left|1-w_{k}\right|}\left(\left|a-w_{k} b\right|+r_{1}+\left|w_{k}\right| r_{2}\right) .
$$

Proof of (4). Let

$$
\begin{aligned}
& F_{1}(z)=(z-\alpha)^{n}-\lambda(z-\beta)^{r} \\
& G(z) \equiv z^{r} \cdot F_{1}\left(\frac{1}{z}\right)=z^{r-n}(1-z \alpha)^{n}-\lambda(1-\beta z)^{r}
\end{aligned}
$$

hence $G(z)$ can also be written in the form:

$$
G(z)=(-\alpha)^{n}(z-\gamma)^{r}-\lambda(1-\beta z)^{r},
$$

where $\gamma$ ranges over a circle including 0 and the points $1 / \alpha$. If $G\left(z_{0}\right)=0$, then $z_{0}=(\delta+\gamma) /(1+\delta \beta)$, where $\delta=\lambda^{1 / r}(-\alpha)^{-n / r}$. Any zero of $F_{1}(z)$ is thus of the form $(1+\delta \beta) /(\delta+\gamma)$. Let $C(a, b)$ denote the circle $|z-a| \leqq b$. If $\alpha \in C\left(a, r_{1}\right)$, then

$$
\frac{1}{\alpha} \in C\left(\frac{\bar{a}}{|a|^{2}-r_{1}^{2}}, \frac{r_{1}}{|a|^{2}-r_{1}^{2}}\right)
$$

and

$$
\gamma \in C\left(\frac{e^{-i \phi}}{2\left(|a|-r_{1}\right)}, \frac{1}{2\left(|a|-r_{1}\right)}\right), \phi=\arg a
$$

Thus 


$$
|\gamma| \leqq\left(|a|-r_{1}\right)^{-1}<|\lambda|^{1 / r}\left(r_{1}+|a|\right)^{-n / r} \leqq|\delta|
$$

by our assumption $d\left(|a|-r_{1}\right)-1>0$.

Now

$$
z_{0} \in C\left(\frac{\beta d^{2}-\bar{\gamma}}{d^{2}-|\gamma|^{2}}, \frac{d|\beta \gamma-1|}{d^{2}-|\gamma|^{2}}\right),
$$

where $d=|\lambda|^{1 / r}\left(r_{1}+|a|\right)^{-n / r}$. Taking into account the inequalities $|\gamma|$ $\leqq\left(|a|-r_{1}\right)^{-1},|\beta| \leqq|b|+r_{2}$, we arrive at (4) after a short calculation.

Proof of (5). From $F_{1}(z)=(z-\alpha)^{n}-\lambda(z-\beta)^{r}$ it follows that

$$
z^{r} F_{1}\left(\frac{1}{z}+\beta\right)=-\lambda+z^{r-n}[1+(\beta-\alpha) z]^{n}
$$

If $F_{1}(\zeta)=0$, then

$$
-\gamma z^{k}+z^{k-1}-\mu=0
$$

with $\zeta=1 / z+\beta, \mu=(\lambda)^{1 / n}, \gamma=\alpha-\beta$.

The left-hand side of (7) can be written in the form

$$
\left(\frac{\gamma}{\mu} z^{k}+1\right)\left(z^{k-1}-\mu\right)-\frac{\gamma}{\mu} z^{2 k-1}
$$

It follows by Szegö's Theorem [3, p. 60] that

$$
\begin{aligned}
|z| & \geqq \frac{1}{2} \operatorname{Min}\left[|\mu|^{1 / k}|\gamma|^{-(1 / k)},|\mu|^{1 / k-1}\right] \\
& =\frac{1}{2} \operatorname{Min}\left[|\lambda|^{1 / r-n},|\lambda|^{1 / r}|\beta-\alpha|^{-n / r}\right]
\end{aligned}
$$

and $|\zeta-\beta| \leqq 2\left\{\operatorname{Min}\left[|\lambda|^{1 / r-n},|\lambda|^{1 / r}|\beta-\alpha|^{-(n / r)}\right]\right\}^{-1}$, (5) follows easily.

It is worthwhile to remark that by the same manipulation we can also obtain a lower bound for the zeros of $f(z)-\lambda g(z)$ namely writing

$$
-\gamma z^{k}+z^{k-1}-\mu z+\frac{\mu}{\gamma}=\left(-\mu z+\frac{\mu}{\gamma}\right)\left(z^{k-1} \frac{\gamma}{\mu}+1\right) .
$$

It follows by the same theorem due to Szegö that all the zeros of $-\gamma z^{k}$ $+z^{k-1}-\mu z$ are in $|z| \leqq 2 \operatorname{Max}\left(1 /|\gamma|,(\mu / \gamma)^{1 / k-1}\right)$. The final estimate is $|\zeta-\beta| \geqq\left\{2 \operatorname{Max}\left[|\alpha-\beta|^{-1},|\lambda|^{1 / n k}|\alpha-\beta|^{-k}\right]\right\}^{-1}$. To obtain a meaningful result it is necessary to suppose that $\operatorname{Min}|\alpha-\beta|>0$; then

$$
\begin{aligned}
& |\zeta-b| \geqq\left\{2 \operatorname { M a x } \left[\left(|a-b|-\left(r_{1}+r_{2}\right)\right)^{-1},\right.\right. \\
& \left.\left.|\lambda|^{1 / r}\left(|a-b|-\left(r_{1}+r_{2}\right)\right)^{-1 / k}\right]\right\}^{-1}-r_{2} .
\end{aligned}
$$


Proof of (6). Write $F_{1}(z)=f_{1}(z)-\lambda g_{1}(z), f_{1}(z)=(z-\alpha)^{n}-\lambda(z-\beta)^{n}$, $g_{1}(z)=(z-\beta)^{r}-(z-\beta)^{n}$.

The zeros of $f_{1}(z)$ are in the union of the circles

$$
C\left(\frac{a-w_{k} b}{1-w_{k}}, \frac{r_{1}+\left|w_{k}\right| r_{2}}{\left|1-w_{k}\right|}\right)
$$

(see, e.g., $[3$, p. 57]); hence in $C(0, r)$.

The zeros of $g_{1}(z)$ are in $C\left(b, r_{2}+1\right)$. Since $f_{1}(z)$ and $g_{1}(z)$ are both of degree $n$ we can use the result in [3] to obtain (6).

We conclude this discussion by proving some results about the location of part of the zeros of the polynomial $(z-\alpha)^{n}-\lambda(z-\beta)^{r}$.

THEOREM 4. At least $n$ zeros of the polynomial $(z-\alpha)^{n}-\lambda(z-\beta)^{r}$ are in the circle

$$
\begin{array}{ll}
|z-\alpha| \leqq \frac{n}{r-n}|\alpha-\beta| & \text { if } n<r \leqq 2 n, \\
|z-\alpha| \leqq|\alpha-\beta| & \text { if } r \geqq 2 n,
\end{array}
$$

and at most $n$ zeros of the above polynomial are in the circle

$$
\begin{array}{ll}
|z-\alpha| \leqq|\alpha-\beta| & \text { if } n<r \leqq 2 n, \\
|z-\alpha| \leqq \frac{n}{r-n}|\alpha-\beta| & \text { if } r \geqq 2 n,
\end{array}
$$

for all complex $\lambda$.

Proof. By a straightforward calculation one obtains that $\operatorname{Re}((z-A) /(z-B))$ $>0(<0)$ if and only if

$$
z \notin C\left(\frac{A+B}{2}, \frac{|A-B|}{2}\right), \quad\left(z \in C\left(\frac{A+B}{2}, \frac{|A-B|}{2}\right)\right)
$$

for $A \neq B$.

Now

$$
\frac{\partial}{\partial \theta} \underset{|z-\alpha|=R}{\arg }\left[\frac{(z-\alpha)^{n}}{-\lambda(z-\beta)^{r}}\right]
$$

$$
=\operatorname{Re}\left[(z-\alpha)\left(\frac{n}{z-\alpha}-\frac{r}{z-\beta}\right)\right]=(n-r) \operatorname{Re}\left[\frac{z+\frac{r \alpha-n \beta}{n-r}}{z-\beta}\right] .
$$

Since $n<r$ it follows that (8) is positive if and only if 
(9)

$$
z \in C\left(\frac{r(\alpha+\beta)-2 n \beta}{2(r-n)}, \frac{r(\alpha-\beta)}{2(r-n)}\right)
$$

In this case

$$
\begin{aligned}
\underset{|z-\alpha|=R}{\arg }\left[(z-\alpha)^{n}\right. & \left.-\lambda(z-\beta)^{r}\right] \\
& =\underset{|z-\alpha|=R}{\Delta \arg }\left[\frac{(z-\alpha)^{n}}{-\lambda(z-\beta)^{r}}+1\right]+\underset{|z-\alpha|=R}{\Delta \arg }\left[(-\lambda)(z-\beta)^{r}\right] \\
& \leqq \underset{|z-\alpha|=R}{\arg }\left[\frac{(z-\alpha)^{n}}{-\lambda(z-\beta)^{r}}\right]+\underset{|z-\alpha|=R}{\arg }\left[-\lambda(z-\beta)^{r}\right]=2 \pi n .
\end{aligned}
$$

Thus if

$$
C(\alpha, R) \subset C\left(\frac{r(\alpha+\beta)-2 n \beta}{2(r-n)}, \frac{r|\alpha-\beta|}{2(r-n)}\right),
$$

then the polynomial $(z-\alpha)^{n}-\lambda(z-\beta)^{r}$ has at most $n$ zeros in the circle $C(\alpha, R)$. It is easy to see that we can take

$$
R=\frac{r-|r-2 n|}{2(r-n)}|\alpha-\beta| .
$$

This proves the second part of the theorem. Similarly

$$
\frac{\partial}{\partial \theta} \arg \left[\frac{(z-\alpha)^{n}}{-\lambda(z-\beta)^{r}}\right]<0
$$

if and only if

$$
z \notin C\left(\frac{r(\alpha+\beta)-2 n \beta}{2(r-n)}, \frac{r|\alpha-\beta|}{2(r-n)}\right)
$$

and we can set

$$
R=\frac{|\alpha-\beta|}{2(r-n)}(r+|r-2 n|) .
$$

It follows in particular that for $r=2 n$, the circle $|z-\alpha| \leqq|\alpha-\beta|$ contains exactly $n$ zeros of the polynomial $(z-\alpha)^{n}-\lambda(z-\beta)^{r}$.

The following theorem generalizes a result due to Biernacki and Jankowski [1], [2].

Theorem 5. Let $P(z)=a_{p} z^{p}+a_{p-s} z^{p-s}+\cdots+a_{0}, Q(z)=b_{q} z^{q}+b_{q-t} z^{q-t}+$ $\cdots+b_{0} . a_{p} b_{q} \neq 0, q>p, s \geqq 1, t \geqq 1$ have all their zeros in the circles $|z|$ $\leqq R_{1}$ and $|z| \leqq R_{2}$, respectively. Let $r=\operatorname{Min}(s, t) \geqq 1$. At least $p$ zeros of the polynomial

$$
P(z)+\lambda Q(z)
$$


are in the circle

$$
|z| \leqq \operatorname{Max}\left\{\left(\frac{q R_{1}^{r}+p R_{2}^{r}}{q-p}\right)^{1 / r}, R_{2}\right\}
$$

Proof.

$$
\underset{|z|=R}{\operatorname{Max}} \frac{d}{d \theta} \arg \frac{P(z)}{Q(z)} \leqq \operatorname{Max}_{|z|=R} \frac{d}{d \theta} \arg P(z)-\operatorname{Min}_{|z|=R} \frac{d}{d \theta} \arg Q(z) .
$$

For $R>\operatorname{Max}\left(R_{1}, R_{2}\right)$ we have:

$$
\begin{aligned}
\operatorname{Max}_{|z|=R} \frac{d}{d \theta} \arg \frac{P(z)}{Q(z)} & \leqq \operatorname{Max}_{|z|=R} \operatorname{Re} \sum_{k=1}^{p} \frac{z}{z-\alpha_{k}}-\operatorname{Min} \operatorname{Re} \sum_{k=1}^{q} \frac{z}{z-\beta_{k}} \\
& \leqq p \underset{|z|=R}{\operatorname{Max}} \operatorname{Re} \frac{z}{z-\alpha}-q \underset{|z|=R}{\operatorname{Min} \operatorname{Re} \frac{z}{z-\beta}} \\
& \leqq p \frac{R}{R-|\alpha|}-q \frac{R}{R+|\beta|},
\end{aligned}
$$

where $\alpha_{k}, \beta_{k}$ are the zeros of $P(z)$ and $Q(z)$, respectively, and the functions $\alpha(z), \beta(z)$ satisfy $|\alpha(z)| \leqq R_{1}^{r} / R^{r-1},|\beta(z)| \leqq R_{2}^{r} / R^{r-1}$. This follows by a recent result due to Walsh [4]. If the $m_{k}, \alpha_{k}$, and $z$ are given with $m_{k}>0$, $\left|\alpha_{k}\right| \leqq A,|z|>A$, and $\sum_{k=1}^{n} m_{k} \alpha_{k}^{l}=0$ for $l=1,2, \cdots, j$, then $\alpha=\alpha(z)$ as defined by the equation

$$
\pi_{k=1}^{n}\left(z-\alpha_{k}\right)^{n}=(z-\alpha)^{n}
$$

satisfies the inequality

$$
|\alpha(z)| \leqq A^{j+1} /|z|^{j}
$$

Under the same conditions except that now $\left|\alpha_{k}\right| \geqq A,|z|<A$, and $\sum_{k=1}^{n} m_{k} \alpha_{k}^{-l}=0$ and $l=1,2, \cdots, j$, we have

$$
|\alpha(z)| \geqq A^{j+1} /|z|^{j} .
$$

In deriving (11) we also notice that

$$
\operatorname{Re}\left(\frac{z}{z-\alpha}\right) \leqq\left|\frac{z}{z-\alpha}\right| \leqq \frac{R}{R-|\alpha|}
$$

and

$$
\operatorname{Re}\left(\frac{z}{z-\beta}\right)=\frac{R(R-r \cos (\theta-\phi))}{R^{2}+r^{2}-2 r R \cos (\theta-\phi)},
$$

with $\beta=r e^{i \theta}, z=\operatorname{Re}^{i \phi}$.

The last expression is an increasing function of $\cos (\theta-\phi)$ and attains its minimum for $\cos (\theta-\phi)=-1$. Hence $\operatorname{Re}(z /(z-\beta)) \geqq R /(R+|\beta|)$. It 
follows now from (11) that

$$
\operatorname{Max}_{|z|=R} \frac{d}{d \theta} \arg \frac{P(z)}{Q(z)} \leqq p \frac{R^{r}}{R^{r}-R_{1}^{r}}-q \frac{R^{r}}{R^{r}+R_{2}^{r}}<0
$$

for $R^{r}>\left(\left(p R_{2}^{r}+q R_{1}^{r}\right) /(q-p)\right)$. It is enough to set

$$
R=\operatorname{Max}\left[\left(\frac{p R_{2}^{r}+q R_{1}^{r}}{q-p}\right)^{1 / r}, R_{2}\right]
$$

which implies $R \geqq \operatorname{Max}\left(R_{1}, R_{2}\right)$.

Now one proves similarly to what has been done in Theorem 4 that

$$
\underset{|z|=R}{\Delta} \arg (P+\lambda Q) \geqq 2 \pi p
$$

which concludes the proof.

It is clear that

$$
R \leqq R^{\prime}=\operatorname{Max}\left[\frac{p R_{2}+q R_{1}}{q-p}, R_{2}\right] .
$$

The estimate $|z| \leqq R^{\prime}$ is due to Biernacki [1]. For large $r, R$ tends to $\operatorname{Max}\left(R_{1}, R_{2}\right)$.

\section{BIBLIOGRAPHY}

1. M. Biernacki, Sur les équations algebriques contenant des paramètres arbitraires, Bull. Acad. Polon. Sci. Sér. A (1927), 541-685.

2. W. Jankowski, Sur les zeros d'un polynomial contenant un paramètre arbitraire, Ann. Polon. Math. 3 (1957), 304-311.

3. M. Marden, The geometry of the zeros of a polynomial in a complex variable, Math. Surveys No. 3, Amer. Math. Soc., Providence, R. I. 1949.

4. J. L. Walsh, A theorem of Grace on the zeros of polynomials, revisited, Proc. Amer. Math. Soc. 15 (1964), 354-360.

5. Mishael Zedek, Continuity and location of zeros of linear combinations of polynomials, Proc. Amer. Math. Soc. 16 (1965), 78-84.

HARVARD UNIVERSITY,

Cambridge, Massachusetts 